\title{
La opacidad del trabajo de enfermería y las configuraciones del riesgo
}

\section{The opacity of nursing work and configurations of risk}

'Doctor en Educación. Profesor Adjunto III, Universidade Federal de Itajubá, campus Itabira. Itabira, Minas Gerais, Brasil. $\triangle$ (iD)

${ }^{2}$ Doctora en Filosofía. Profesora Asociada, Universidade Federal de Minas Gerais. Belo Horizonte, Minas Gerais, Brasil. $\triangle$ iD
Davidson Passos Mendes ${ }^{1}$, Daisy Moreira Cunha²

RESUMEN El artículo tiene por objetivo revelar la invisibilidad del trabajo de enfermería y las estrategias individuales y colectivas instauradas como posibilidades de regulación del personal de enfermería frente al riesgo laboral de asistir al paciente psiquiátrico en situación de crisis. Desde un enfoque teórico metodológico que combinó el análisis ergonómico del trabajo con aportes de la ergología, se investigó y analizó, en 2012, a un equipo del centro de atención de crisis de un hospital público psiquiátrico brasileño, compuesto por 17 enfermeros y dos médicos (director y coordinador del sector). Los resultados revelaron que la asistencia a los usuarios está fuertemente caracterizada por el trabajo en equipo, especialmente en la confrontación de saberes del personal médico y de enfermería, mediada, muchas veces, por la intervención de otros trabajadores como los asistentes sociales, el personal administrativo y el portero. Es decir, el equipo se extiende más allá del cuerpo asistencial involucrando también al servicio de apoyo. Se evidenció también que esa construcción se efectúa en el día a día del trabajo y requiere competencias para la cooperación, además del desarrollo de habilidades colectivas.

PALABRAS CLAVES Enfermería Psiquiátrica; Riesgos Laborales; Exposición Profesional; Brasil.

ABSTRACT The article aims to reveal the invisibility of nursing work and the individual and collective strategies established to face the labor risks of attending psychiatric patients in situations of crisis. The theoretical-methodological approach combined ergonomic analysis with ergology contributions, examining in 2012 a team from an emergency unit of a Brazilian public psychiatric hospital composed of 17 nurses and two physicians (the director and medical coordinator of the unit). The results revealed that user care is strongly characterized by teamwork, especially in the confrontation of the knowledge of doctors and nurses, and is often mediated by other professionals such as social workers, administrative staff, and entrance guards. In this way, the health team extends beyond the care staff to include support services as well. It was also evidenced that this construction is effective in day-to-day work and requires skills for cooperation as well as the development of collective abilities.

KEY WORDS Psychiatric Nursing; Occupational Risks; Occupational Exposure; Brazil. 


\section{INTRODUCCIÓN}

Este artículo se basa en una investigación mayor titulada $O$ agir competente como estratégia de gestão do risco de violencia no trabalho: o ponto de vista da atividade humana de trabalho dos técnicos de enfermagem de uma instituição pública psiquiátrica $^{(1)}$, realizada en un hospital público de emergencia psiquiátrica, y centrada en el riesgo y los agravios de la asistencia al paciente psiquiátrico en crisis. Se sitúa en el campo de la producción de conocimientos para el desarrollo de criterios ergonómicos y ergológicos destinados a la concepción y gestión de sistemas de trabajo en el sector salud, que incluyan las condiciones de desempeño laboral y las repercusiones de las condiciones de ejecución del trabajo sobre la salud de la población trabajadora involucrada en la asistencia en salud mental, cuyo espacio y técnica están en profunda transformación estructural.

\section{La paradoja de las normas: entre la necesidad y la insuficiencia}

De acuerdo con Maciel y Telles ${ }^{(2)}$, la práctica de evitar riesgos siempre estuvo asociada a la posibilidad de ocurrencia de eventos indeseables. Sin embargo, "riesgo" es un término muy reciente y su definición conceptual está lejos de reunir consensos ${ }^{(3,4)}$. Vivir nos obliga, necesariamente, a tener en cuenta los riesgos. Protegerse de la muerte, de la enfermedad, de la tempestad, del fracaso en los negocios, no es una estrategia reciente ${ }^{(3)}$. Es una noción genuinamente moderna y está implicada en la reorientación de las relaciones que los individuos y las colectividades establecen con los acontecimientos que pueden ocurrir, cuya idea central es controlar el futuro. Surge en oposición al concepto de fatalidad y destino, asociado a una cierta contingencia o ambigüedad derivada de las diversas dinámicas del mundo social.

Según Mazet y Guilhermain ${ }^{(5)}$, "...el riesgo caracteriza la eventualidad de un evento (o de una situación) no deseada (o temida) y sus efectos o consecuencias". Se trata de una medida del nivel de peligro, concepto cualitativo que expresa una potencialidad, una condición o situación física con potencial de tener consecuencias no deseadas, como la muerte o las lesiones corporales, por su probabilidad de ocurrir, su gravedad y su aceptabilidad ${ }^{(6)}$.

El concepto de riesgo posee, por lo tanto, tres componentes básicos: 1) su potencial de pérdidas y daños; 2) la incertidumbre de las pérdidas y daños; 3 ) la relevancia de las pérdidas y daños. De esta forma, el riesgo es igual a la probabilidad de daños multiplicado por la magnitud de las consecuencias en función del tiempo ${ }^{(7)}$. Los avances tecnológicos, científicos y del patrón productivo contribuyeron a esa nueva percepción del riesgo asociado a cambios en la propia naturaleza del riesgo derivado.

Como caracterización del objeto de estudio, en este contexto, la enfermería es una práctica históricamente estructurada, constituida por diferentes maneras de cuidar, determinadas por las relaciones sociales de cada momento histórico ${ }^{(8)}$.

Actualmente, el trabajo de enfermería forma parte del trabajo colectivo en salud, es especializado, está dividido y jerarquizado entre auxiliares, técnicos y enfermeros de acuerdo con la complejidad de concepción y ejecución. Aunque detenta una autonomía relativa en relación con los demás profesionales, la enfermería está subordinada al gerenciamiento del acto asistencial en salud, ejecutado por los médicos ${ }^{(8,9)}$.

Según Almeida ${ }^{(10)}$, la enfermería es parte del proceso de asistencia y del trabajo médico y su acción es un instrumento de trabajo médico que "cuidará" o "hará cuidar" del cuerpo enfermo. La observación en enfermería, el relevamiento de datos, la planificación, la revolución, la evaluación de los pacientes, los sistemas de asistencia, los procedimientos técnicos y de comunicación e integración entre pacientes y enfermería y entre los diversos profesionales son, entre otros, algunos de los instrumentos de trabajo de este proceso de "cuidar", con la misma finalidad del trabajo médico, que es "curar cuerpos individuales". 
La autora también revela, como resultado histórico y social, otro tipo de actividad de enfermería que es "administrar" (que no es realizada por todas las categorías de enfermería, sino por la enfermera), cuyos instrumentos son los propios modelos y métodos de la administración: normas y rutinas, la fuerza de trabajo en enfermería (los auxiliares), los equipamientos y materiales permanentes $y$ aquellos para la manipulación y administración de drogas y soluciones.

En el ámbito de la enfermería, el trabajo se caracteriza por agrupar factores que pueden representar riesgos para la salud de sus trabajadores. Diversos estudios ${ }^{(11,12,13)}$ han mostrado que la exposición a factores de riesgos mecánicos ${ }^{(14)}$ y ambientales; al trabajo nocturno ${ }^{(15)}$, a la manipulación de productos químicos ${ }^{(16)}$, a cargas horarias extensas asociadas a las dobles jornadas ${ }^{(17)}$, a la radiación ionizante, al excesivo peso durante la asistencia al paciente ${ }^{(18)} \mathrm{y}$ al contacto directo con material infectado ${ }^{(19)}$ se agrava aún más por los recursos materiales insuficientes e inadecuados, que ocasionan condiciones inseguras de trabajo ${ }^{(8)}$.

Existen también factores psicosociales del trabajo de enfermería que provocan un aumento de los índices de ausentismo y padecimientos en los trabajadores. Según Manetti ${ }^{(20)}$, los factores psicosociales presentes en el trabajo de enfermería están relacionados con cambios e innovaciones en la organización del trabajo (autonomía, clima organizacional, oportunidad de crecimiento profesional) y con violencia, cuyas consecuencias provocan estrés, bajo nivel de satisfacción en el trabajo, desgaste físico-mental, sufrimiento, ausentismo y rotatividad (21).

La normatización está asociada a la burocratización cada vez mayor de las diversas actividades sociales, y se ha mostrado útil, aunque haya sido también criticada por el hecho de que el paradigma burocrático no capta siempre la evolución y las situaciones imprevisibles a nivel organizacional. A través de las normas se intentan controlar los procedimientos comunes, la propia emoción es racionalizada desde una perspectiva que aferra a los sujetos a procedimientos prescritos.
La distancia entre la burocratización y la realidad del trabajo es también un hecho. El papel central desempeñado por la racionalidad técnica, en este irresistible y, a veces, arriesgado camino, combina siempre método científico y procedimientos. Por lo tanto, la normatizaciones nos obligan a estar alertas y vigilantes en busca de un constante compromiso con las reglas y los reglamentos.

Las reglas y los procedimientos son fundamentales para el funcionamiento de una organización moderna, resultan necesarios para la gestión de la seguridad, al intentar rastrear, controlar y anticipar las actividades sociales. La normatización captura dos fases que están totalmente incorporadas: por un lado, el control y, por otro, la explicitación. No es una novedad que los procedimientos escritos no solo indican cómo deben ser realizadas las tareas, sino que funcionan también como mecanismos restrictivos y controladores de los trabajadores con relación a aquello que debe ser hecho y cómo está siendo realizado. Permiten, también, la expresión formal de las prácticas de trabajo para materializar, divulgar y abrir la discusión desde las bases con los niveles de la organización, los colaboradores externos y quienes regulan las prácticas, por ejemplo.

Según Bourrier ${ }^{(22)}$, sociólogos y ergonomistas han mostrado hace mucho tiempo que los procedimientos y las reglas generan restricciones, pero también resultan necesarios como elementos de seguridad y control frente a las prácticas inseguras, las reglas insuficientes y o gerenciamientos inconsistentes. Para la autora, la norma puede aportar confort a las personas y reducir la ansiedad con relación a lo nuevo y lo incierto. Muchas veces puede ser necesario ir más allá de las reglas para alcanzar los objetivos, pero la norma puede servir también como directriz cuando el curso de la acción no se ha vivenciado anteriormente y resulta controvertido. La normatización legitima la racionalidad técnica ${ }^{(6)}$.

Desde el punto de vista situado, las normas se han mostrado insuficientes para dar cuenta de las imprevisibilidades y variabilidades existentes en la actividad del trabajo de enfermería del hospital psiquiátrico 
analizado. Qué se hace y cómo se hace está invisibilizado y es desconocido por los gestores del sistema, pero es esencial para la construcción de la seguridad local (tanto de los trabajadores como de los pacientes atendidos). Por lo tanto, es necesario que emerjan las competencias individuales y colectivas construidas y valoradas en el contexto, por medio de métodos que hagan aflorar estas microrregulaciones y que puedan ser captadas para hacer evolucionar las normas y producir seguridad.

Boyé y Robert ${ }^{(23)}$ definen competencia como "conjuntos de saber-hacer requeridos por las tareas, vinculados a las personas que los ponen en práctica, reconocidos como tales por el ambiente en el cual son ejercidos, directamente dependientes del contexto sociotécnico y cultural de aplicación". Leplat y Montmollin ${ }^{(24)}$, en un intento de conceptualizar competencia, la definen como: "conjuntos estabilizados de saberes y de saber-hacer, de conductas tipo, de procedimientos patrón, de tipos de raciocinio que se pueden aplicar sin un nuevo aprendizaje". Según los autores, las competencias sedimentan y estructuran las adquisiciones de la historia profesional, permiten anticipar los fenómenos, lo implícito de las instrucciones y la variabilidad de las tareas.

Las competencias permiten desarrollar y utilizar estrategias para explorar los conocimientos o recursos cognitivos y sociales para una determinada acción. Esta exploración consiste en preparar la acción, o sea, combinar los conocimientos adquiridos, seleccionar y orientar la acción (planificación, distribución, realización, control), como también combinar conocimientos derivados de la experiencia pasada con expectativas racionales sobre eventos futuros ${ }^{(25)}$. Estas mediaciones refrendan que se trata de seres vivos humanos actuando en medios afectados por múltiples (micro) variabilidades ${ }^{(26)}$.

Según Schwartz"(27), "el trabajo es un lugar de acontecimientos complejos", o sea, una unidad problemática entre la actividad humana, las condiciones reales de trabajo y los resultados efectivos obtenidos. En ese sentido, la perspectiva ergológica considera la actividad "siempre como un encuentro [...] de una realidad siempre singular"(28), como un debate de normas (normas antecedentes y renormalizaciones) orientadas por valores y traducidas como la síntesis operativa que envuelve las diversas dimensiones inseparables de la vida, que se generan en situaciones concretas y que tornan visible el drama de la actuación humana de forma global, en el que la actividad es una dimensión general antropológica ${ }^{(29,30)}$.

De acuerdo con Schwartz ${ }^{(31)}$, es necesario abordar el trabajo como un vaivén permanente entre las condiciones ambientales objetivables, que exponen a los riesgos anticipables, denominados por el autor como "riesgos profesionales" y una dimensión enigmática, que en parte reconfigura la actuación humana en el trabajo y conduce a lo que el autor Ilama "riesgos del trabajo". Según Echternacht ${ }^{(32)}$, "trabajar es gestionarse en un medio circunscrito por normas de orden técnico, organizacional, gerencial, por entre estructuras productivas que heterodeterminan los objetivos del trabajo humano, sus instrumentos, su tiempo, su espacio". Pero tales niveles de heterodeterminación no excluyen la actividad humana en su movilización de saberes y valores incorporados a las prácticas, condición para actuar de forma competente.

Si partimos del presupuesto ergonómico de que el trabajo real es diferente del prescrito, la ergología, añade que no solo es imposible, sino que es "invivible" reproducir completamente las normas ${ }^{(33)}$. De modo que, para gestionar las variabilidades, es preciso hacer "uso de sí, de las propias capacidades, de los propios recursos y elecciones", lo que se configura en una "drama del uso de sí". Según Schwartz y Durrive, "trabajar es arriesgarse, hacer 'uso de sí'"(33). Según Cunha ${ }^{(26)}$, "incluso, obedecer normas preexistentes ya es elegir. Y al operar en los intersticios de las normas preexistentes, el hombre hace historia". Tales elecciones determinan "la relación con los otros o con el mundo en el cual se quiere vivir"(33). $^{\prime \prime} \mathrm{Si}$, por un lado, trabajar es siempre un proceso de singularización y resingularización, por otro, las elecciones están 
siempre atravesadas por la dimensión colectiva, entidad cuyos contornos variables se constituyen espontáneamente "con relación a, o en relación con, la organización"(33).

Si en la actividad hay un crisol de patrimonios constituido principalmente de competencias y de "saberes-hacer", la renormatización es un proceso de invención de soluciones localizadas y, luego, consolidadas y transmitidas para la eficacia y para la salud $^{(34)}$. Según Nouroudine ${ }^{(34)}$, las zonas de desarrollo de la acción, de la actividad, de las representaciones, de la memoria, de la consciencia, del inconsciente, podrían vincularse entre sí en la instancia denominada cuerposí. Para Schwartz ${ }^{(35)}$ :

El cuerpo-sí es historia, historia como memoria sedimentada, organizada en la miríada de los circuitos de la persona; pero también historia como matriz, energía productora de lo inédito: en la medida que la ambición renormatizante es al mismo tiempo impuesta al ser [...] y también demandada como exigencia de vida, como su propia apelación de salud, instrumentándolo sin cesar para intentar transformar lo que es objetivamente un medio [...] en aquello que podría constituir su medio.

En este sentido, las actividades humanas son el lugar de compromiso de quienes son sus autores. Para Echternacht ${ }^{(32)}$, arbitrar entre "el uso de sí por sí mismo y el uso de sí por otros" implica renormatizaciones, en que las normas preexistentes se reinterpretan con el objetivo de reajustarlas para sí mismos y para la situación presente.

\section{ASPECTOS METODOLÓGICOS}

El abordaje metodológico utilizado en este estudio se basó en el análisis ergonómico ${ }^{(36)}$ y ergológico ${ }^{(37)}$ del trabajo. Se trata de un método cualitativo-descriptivo, con metodología y herramientas de análisis pertinentes para identificar los principales factores técnicos y organizacionales que intervienen en la gestión de la salud en el trabajo contextualizado y gestionar, en conjunto con los demandantes, proposiciones para la adecuación preventiva de los actuales sistemas sociotécnicos y organizacionales que aplicamos en el análisis del trabajo hospitalario.

Los procedimientos metodológicos de este estudio se realizaron conforme la Resolución 466/2012 del Consejo Nacional de Salud y fueron aprobados por los Comités de Ética en Investigación de la Universidade Federal de Minas Gerais (CAAE: 0492.0.203.287-11) y de la Fundação Hospitalar de Minas Gerais (096-B/2011).

El objetivo del artículo es revelar la invisibilidad del trabajo de enfermería y las estrategias individuales y colectivas instauradas como posibilidades de regulación de los técnicos de enfermería, frente al riesgo laboral de la asistencia al paciente psiquiátrico y discutir el papel normativo en el contexto analizado.

\section{Relevamiento y análisis de los datos}

La recolección de datos en campo se realizó en 2012, en varios momentos y en distintas situaciones, sumando un total de 244 horas. En un primer momento, se analizó la demanda y los aspectos técnicos y organizacionales del hospital y se delimitó el sector. La selección se realizó por medio de observación y entrevistas de autoconfrontación con los auxiliares y técnicos de enfermería del sector y el análisis del flujograma de entrada del paciente al sistema, el cual mostró este sector como un lugar importante para la configuración del proceso de trabajo y de gestión de los riesgos en los otros sectores de la institución.

En esta etapa, participaron los médicos involucrados (director clínico del hospital y coordinador del lugar analizado), se buscó mapear las demandas y relacionarlas con los sistemas de trabajo, apuntando a la comprensión de la singularidad de los contextos productivos en foco. A partir de allí se delimitó el sector y la población a ser analizada. Los criterios utilizados para la elección fueron: 1) gravedad, en cuanto al potencial de riesgo; 2) centralidad, 
en cuanto a las condiciones para el desempeño global del sistema; 3) accesibilidad, en cuanto a las condiciones para el análisis.

Participaron 19 trabajadores: 17 auxiliares y técnicos de enfermería de un sector de emergencia y 2 médicos (director y coordinador médico) del centro de atención de crisis de un hospital público psiquiátrico. Se realizaron entrevistas abiertas y semiestructuradas; observación general de las actividades de trabajo por medio del método del análisis ergonómico del trabajo y recolección de datos relativos a la distribución temporal y espacial de las actividades. Con el recorte y el foco establecido, se inició un análisis detallado de las situaciones críticas identificadas y el relevamiento de las tareas (objetivos de producción, métodos, normas de productividad y calidad), exigencias operativas, de interacción, de comunicación y de cooperación. Luego del relevamiento de las tareas, se hicieron observaciones sistemáticas de las actividades en situaciones reales de trabajo y registro de verbalizaciones (simultáneas y consecutivas) por medio de entrevistas de autoconfrontación, y el método de análisis ergonómico del trabajo.

Mediante lo observado y validado durante el análisis ergonómico del trabajo se revelaron los principales elementos que condicionaban y modificaban los modos operativos y los valores del contexto, y que muchas veces pueden facilitar $y$, otras, dificultar las configuraciones colectivas, de las estrategias de gestión de riesgos o de los procesos de aprendizaje y su actualización necesaria para la gestión de riesgos en ciertas situaciones. Luego del análisis de los datos y las conclusiones diagnósticas se definieron criterios para la transformación preventiva de las situaciones.

Como el objetivo no era imponer el análisis, los conocimientos generados en el trabajo se pusieron en discusión en diversos momentos y situaciones. Algunas discusiones se dieron en forma individual con alguno de los participantes y, otras, en grupo. El material de confrontación, recogido en la observación de situaciones, filmaciones y entrevistas propiciaron cuestionamientos sobre las acciones y comunicaciones, sacando a la luz los saberes, los valores situados en el contexto, las intencionalidades y las limitaciones propias de las (im)posibilidades del cumplimiento de las reglas.

Las discusiones grupales con los técnicos de enfermería se realizaron en el puesto de enfermería, en los momentos de menor demanda de asistencia, de forma que pudieran participar y, al mismo tiempo, gestionar el proceso de trabajo. Estas discusiones se intentaron realizar en otros momentos y espacios, pero tuvieron baja adhesión. Los resultados también se presentaron, discutieron y validaron con los gestores locales (coordinación médica y supervisión de enfermería) por medio de la devolución y discusión de los datos revelados y presentación del material escrito. Al enviar el material escrito a la dirección y a los demás gestores para su lectura, se pudo percibir una baja adhesión y retorno de respuestas. Un camino construido que se mostró más eficiente fue el de presentar los datos de forma expositiva y oral.

La validación del análisis del trabajo se estructuró de manera de ayudar a construir los recorridos, establecer y direccionar el foco de observación y confirmar/rechazar las hipótesis formuladas hasta entonces. Los resultados del análisis del trabajo constituyen, de este modo, la materia prima de los compromisos que serán tomados por los actores involucrados que, a su vez, son autores de ese proceso, participan de la construcción de la historia.

Para comprender la actividad, que es más global que la acción, no es suficiente el acto de realizar una tarea, es necesario también articular el sentido, restringir la mirada, enfocar, ya que la actividad está compuesta, además, por su entorno no evidente. De esta forma las actividades suspendidas, cuestionadas o impedidas y sus contraactividades ${ }^{(7)}$ fueron admitidas en el análisis. Este posicionamiento generó una reflexión conjunta del analista-investigador con los protagonistas que participaron y ayudaron a la reconstrucción de sentidos para la actividad.

El análisis del trabajo con su validación colectiva modifica el nivel y la naturaleza de la contribución de los diversos actores involucrados, posibilita tomar decisiones en conjunto, al mismo tiempo que desestabiliza 
posiciones en las cuales los diferentes actores se encuentran aislados.

En el análisis de los resultados y la validación se observó que mucho de lo que se hace en aquel contexto productivo es "invisible" para los gestores del sistema, aunque ya se haya reconocido como necesaria una cierta "experticia" para lidiar con el paciente en el centro de atención de crisis. De esta forma, el conocimiento producido por el análisis de las situaciones concretas no puede ser separado del uso que se puede hacer por parte de aqueIlos que contribuyen a la elaboración de ese conocimiento, sobre todo, porque a partir de este posicionamiento se puede viabilizar otro modo de regulación, de editar reglas diferentes, y tener una nueva mirada sobre la estructuración del trabajo. Este espacio de restitución de resultados y validación puede ser un territorio posible para situar las diversas perspectivas de las relaciones de trabajo y su regulación y esclarecer los diferentes "puntos de vista" observados en los estudios sobre el trabajo.

\section{ANÁLISIS DE LA ACTIVIDAD DE ENFERMERÍA EN EL CONTEXTO DEL CENTRO DE ATENCIÓN DE CRISIS: RESULTADOS}

El centro de atención de crisis ocupa una posición fundamental en esta institución, es el locus importante y necesario para la gestión de las camas y de los riesgos de violencia en el hospital. El papel del centro de atención de crisis en la estructura organizacional del hospital, compartido por los diversos grupos profesionales (médicos, enfermeros, auxiliares y técnicos de enfermería, etc.) es el de recibir a los pacientes en crisis, evaluar, tratar, y hacer el pase a las salas (cuando el caso demanda mayor permanencia) y vincularse con los servicios sustitutivos de la red de salud mental, luego del alta.

Cuando se indica la continuidad del tratamiento del paciente, luego de la permanencia en el centro de atención de crisis, pasa a la internación en la sala. En el hospital hay cuatro salas, la $1^{\mathrm{a}}$ y $2^{\mathrm{a}}$ son para mujeres, con
21 camas cada una, y la $3^{\mathrm{a}}$ y $4^{\mathrm{a}}$ para varones, con 30 camas cada una. Hay, también, una unidad clínica de cuidados intermediarios $(\mathrm{UCCl})$, con seis camas, para la permanencia de pacientes inestables clínicamente. Las camas de la UCCI son de retaguardia, cuando se utilizan se reserva una cama en la sala. En el hospital hay, por lo tanto, seis camas en el centro de atención de crisis, 42 en las salas para mujeres, 60 para varones y seis en la $\mathrm{UCCl}$, es decir, un total de 108 camas.

El flujo del paciente en crisis, según la estructura organizacional descrita en la Figura 1, y su permanencia necesaria para estabilizar el cuadro clínico es de extrema importancia, por lo que el centro de atención de crisis es un sector estratégico para la gestión de camas, de riesgos para el propio paciente y terceros y para prescribir la clínica. Sin embargo, se observó una demanda cada vez mayor de las internaciones compulsivas en este hospital y, por esta razón, las estrategias de gestión de camas y de riesgos se vieron comprometidas.

\section{Las transformaciones sociales y los cambios significativos en el perfil de los pacientes: la cuestión del riesgo}

Las transformaciones sociales produjeron cambios significativos en el perfil de los pacientes. Al momento de realizar la investigación, los "drogadictos" componían el $65 \%$ de la demanda del hospital. Los pacientes que accedían al servicio eran, en su mayoría, pacientes tildados de "difíciles" por otras instituciones y que, por lo tanto, no se vincularon a otros servicios. La mayoría de las entradas eran involuntarias y de pacientes en crisis, lo que aumentaba sustancialmente el riesgo en la puerta de entrada, principalmente, a la violencia física y psíquica.

La violencia que envuelve al paciente psiquiátrico, instaurada culturalmente y que circunda todo el entramado social, trae consecuencias sobre la visión que se tiene de esta población. El paciente psiquiátrico es estigmatizado, tratado como criminal o vagabundo, y engloba al usuario de drogas y su exclusión social. La propia violencia 


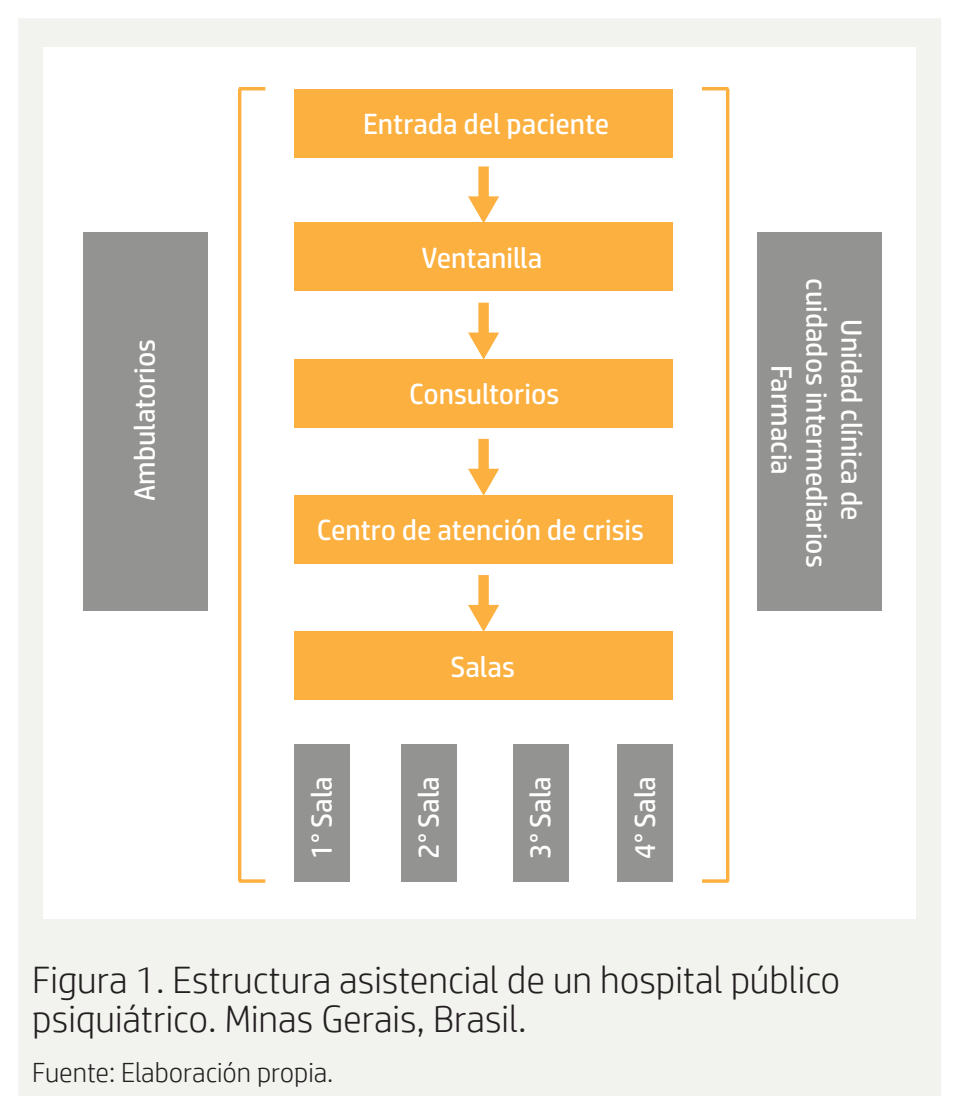

relacionada con la internación se justifica como una respuesta demandada por el Estado a la psiquiatría y legitimada por los familiares. La violencia se instaura y remite a un fin que yace en el futuro: perspectiva terapéutica que apunta al "bien" del sujeto o que objetiva una armonización social.

Foucault, en Historia de la locura en la edad clásica ${ }^{(38)}$, ya advertía sobre el encasillamiento del "enloquecer", las clasificaciones y terapéuticas "científicas" y la consecuente sumisión de la singularidad a la norma de la razón y de la verdad de la mirada psiquiátrica. Según Foucault, se estructura, una red de biopoderes y de disciplinas que conforman el control social del loco.

Muchas veces los psiquiatras sufren presiones (del Estado, la justicia, la policía, la sociedad, la familia) para internar a los pacientes, como fue relatado por un psiquiatra del hospital, entrevistado:
El gobierno tiene la intención de esconder a esta población de usuarios de drogas, especialmente, de crack. Mientras estos pacientes no incomoden a la sociedad, van actuando ahí, siendo escondidos, colocados en los hospitales psiquiátricos. Pero eso se está tornando una calamidad pública, ¿no? Por ejemplo, nosotros estamos con un paciente aquí hoy en nuestro CAC [centro de atención de crisis], no voy a citar su nombre para no complicar a la persona, pero la demanda aquí es que además de ser usuario de crack, él venía robándole objetos a la propia madre, amenazando a familiares... Un paciente que ya viene pasando por el centro de salud, por unidades terapéuticas desde hace tiempo, ¿sí?, de forma involuntaria o voluntaria. El voluntarismo que funciona, si realmente existe, es el del paciente que incluso se acaba de ir del CAC, hace casi media hora. Porque para 
él no era necesario permanecer aquí con nosotros más de 24 horas. Para observar ese período de abstinencia es necesario permanecer por un período mayor de 24, 48, 72 horas ¿sí?, por su seguridad, y él estaba aquí desde el inicio de la guardia abordándonos, abordando a otros del servicio social, con la expectativa de tener hoy el alta, de que la madre venga. La madre ya había estado aquí ayer hablando de la dificultad de manejarlo, hablando de las orientaciones de las otras personas, "¿por qué no abandona?". Y ella: "para mí es difícil". Incluso siendo ella la víctima ¿no? Siendo objeto de él en una situación de esas. Porque ser madre es eso, ¿no? Es dar soporte. Entonces, el proyecto de vida de ella es salir de esa casa donde hay otros familiares y alquilar un lugar donde ella pueda vivir sola con él, incluso con ese hijo estando en esa situación que roba dentro de la propia casa para el consumo de drogas. La familia presiona para la internación, para respirar, pero al mismo tiempo la madre, además de soportar la situación, quiere cuidar, revertir el cuadro y el paciente quiere salir, se exacerba la violencia e incluso se escapa.

En este hospital, el principal criterio de internación involuntaria o compulsiva del paciente es la noción del riesgo de violencia contra sí y contra otros. Los pacientes que llegan, traen consigo, insignias propias del estigma social: "pacientes agitados, agresivos, alcoholizados, peligrosos, presidiarios, pacientes que ya mataron y pueden matar" (Técnico de enfermería 5, Centro de atención de crisis). Se percibe también la violencia en las técnicas de control por parte de la policía, del Servicio de Atención Médica de Urgencia (SAMU) y de la familia.

La violencia social hacia el paciente repercute en la asistencia e instaura una condición de riesgo de violencia para la población trabajadora del hospital psiquiátrico, que ocupa el locus (la puerta de entrada) de la manifestación de la violencia. El paciente "difícil", de internación compulsiva o involuntaria, potencialmente, es un riesgo para sí y va a exponer a otras personas a esta condición de riesgo. Cabe a la población trabajadora, especialmente los técnicos de enfermería, gestionar el objetivo formativo (la violencia y el cuidado con el sujeto/paciente). Como mencionó un psiquiatra:

...el paciente que Ilega, está al límite. Lo traen porque está totalmente desintegrado, agresivo, perdido. La mayoría de las veces, lo trae la policía. La familia ya no aguanta más. Ya tiene un montón de contravenciones, por hacer disturbios en la ciudad, robándole a la familia, agrediendo. $Y$ agrede en serio. Incluso nosotros, como personal de asistencia, hemos sufrido muchas agresiones, violencia física y psíquica (amenazas de que nos van a "agarrar" afuera). Tenemos que cuidar al sujeto que sufre y de nosotros mismos que también sufrimos.

Se observa que decisiones supuestamente diagnósticas responden a una demanda social de control y normalización en las que hay un manejo poblacional realizado por la psiquiatría. Es en este conjunto simbólico que la práctica y los saberes psiquiátricos se tornan visibles en el hospital psiquiátrico, que concretiza la metáfora de la exclusión producida en la modernidad en función de la diferencia.

\section{Opacidad y densidad del trabajo de los técnicos de enfermería}

El trabajo de asistencia a los usuarios del centro de atención de crisis está fuertemente caracterizado por el trabajo en equipo, especialmente en la confrontación de saberes de los médicos y técnicos de enfermería, mediados muchas veces por otros trabajadores como los enfermeros, los asistentes sociales, el personal administrativo y el portero, o sea, el equipo supera al cuerpo asistencial e incluye también al servicio de apoyo.

La transformación de una población de diferentes formaciones y distintas historias de 
vida y realidades en un equipo cohesionado no es un proceso rápido y fácil. Esta construcción se hace efectiva en el trabajo cotidiano y requiere competencias para la cooperación, además del desarrollo de habilidades colectivas. De allí la importancia del aprendizaje a partir de la experiencia, tanto de las vivencias diarias del trabajador en el centro de atención de crisis, como del intercambio con los demás integrantes del equipo, promoviendo una retroalimentación de la experiencia y fortalecimiento del colectivo.

Al observar las actividades de los técnicos de enfermería del centro de atención de crisis, emergieron diversas interacciones necesarias para el intercambio de información, los traslados en busca de medicamentos y la derivación de pacientes a las salas, el relevamiento de datos del sistema y de las planillas de las salas y los diversos momentos de interrupción, como atender el teléfono o asistir a un paciente más urgente que llega y demanda una atención más inmediata: se rompe así, aunque momentáneamente, una acción en curso. Las interacciones son recurrentes, lo que permite afirmar que la actividad supera la visión funcionalista de la tarea y permite la gestión del flujo de información en el servicio.

La Figura 2 y la Tabla 1 son el resultado de la observación del trabajo de un técnico de enfermería del centro de atención de crisis, con una experiencia de 30 años de trabajo en la institución, y revela las diversas actividades realizadas durante 84 minutos que no son conocidas por la estructura organizacional: para los gestores locales esas estrategias de regulación son "invisibles".

Cerca del $51 \%$ del tiempo mensurado fue utilizado para discutir casos con el colectivo de trabajo (médicos, equipo de enfermería, enfermero y otros técnicos, asistente social y personal administrativo encargado del ingreso). El tiempo restante se utilizó en traslados entre sectores, atención a los pacientes, consulta de datos y registro de tareas, organización de las historias clínicas y solicitud de recursos a otros sectores. Hay, incluso, un $12 \%$ de las actividades que fueron asignadas como "otros" en función de la diversidad de acciones realizadas, como: atender

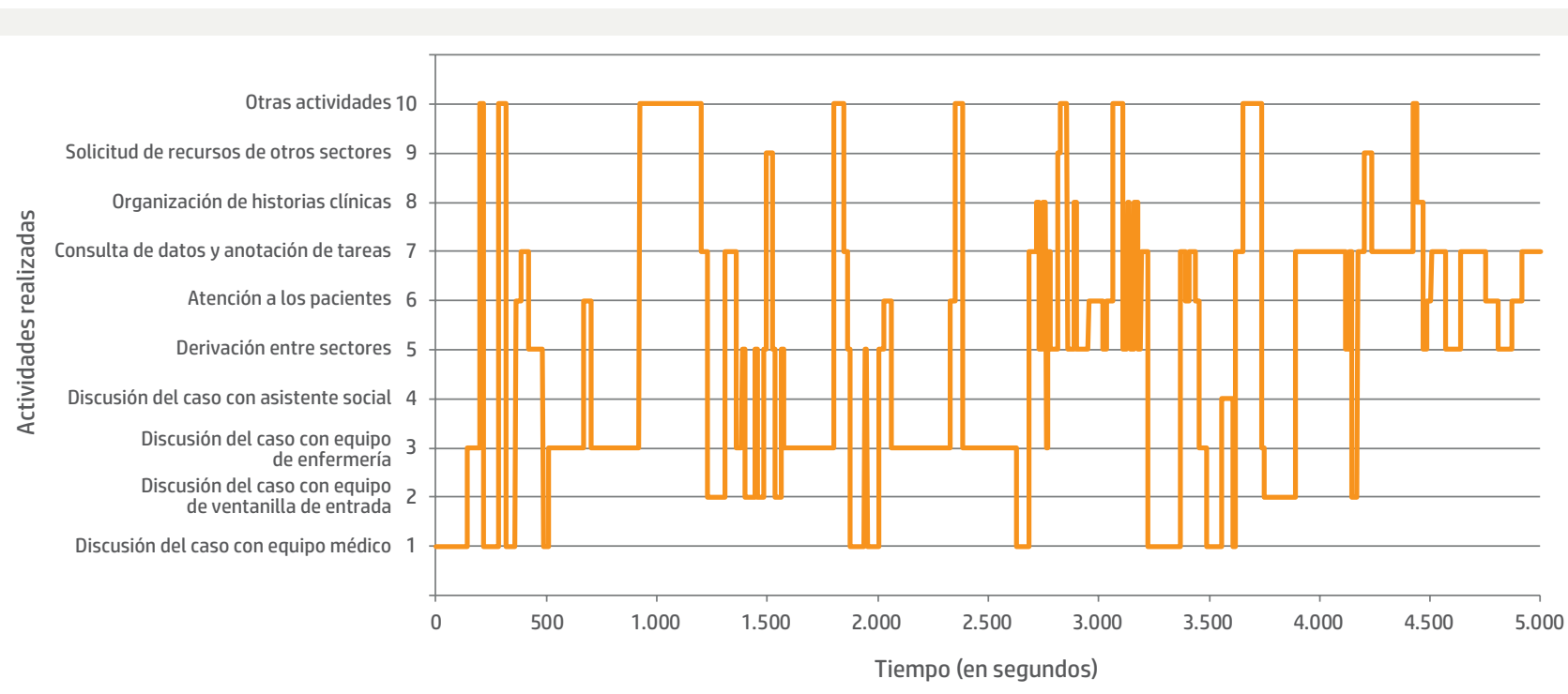

Figura 2. Actividades realizadas durante 84 minutos por un técnico de enfermería del centro de atención de crisis. Minas Gerais, Brasil. 
Tabla 1. Actividades realizadas por un técnico de enfermería del centro de atención de crisis. Minas Gerais, Brasil.

\begin{tabular}{|c|c|c|c|}
\hline \multirow{2}{*}{ Actividades } & \multicolumn{3}{|c|}{ Tiempo analizado } \\
\hline & Segundos & Minutos & $\%$ \\
\hline $\begin{array}{l}\text { Discusión del caso con el } \\
\text { equipo médico }\end{array}$ & 680 & 11,33 & 13,60 \\
\hline $\begin{array}{l}\text { Discusión del caso con el } \\
\text { equipo de la ventanilla de } \\
\text { entrada }\end{array}$ & 346 & 5,77 & 6,92 \\
\hline $\begin{array}{l}\text { Discusión del caso con el } \\
\text { equipo de enfermería }\end{array}$ & 1513 & 25,22 & 30,27 \\
\hline $\begin{array}{l}\text { Discusión del caso con } \\
\text { asistente social }\end{array}$ & 54 & 0,9 & 1,08 \\
\hline Derivación entre sectores & 537 & 8,95 & 10,74 \\
\hline Atención a pacientes & 376 & 6,27 & 7,52 \\
\hline $\begin{array}{l}\text { Consulta de datos y anotación } \\
\text { de tareas (cuaderno de } \\
\text { enfermería, sistema SIGH, y } \\
\text { planilla de gestión de camas) }\end{array}$ & 736 & 12,27 & 14,72 \\
\hline $\begin{array}{l}\text { Organización de historias } \\
\text { clínicas }\end{array}$ & 94 & 1,57 & 1,88 \\
\hline $\begin{array}{l}\text { Solicitud de recursos de otros } \\
\text { sectores }\end{array}$ & 70 & 1,17 & 1,40 \\
\hline Otros & 593 & 9,88 & 11,86 \\
\hline Total & 4999 & 83,33 & 100,00 \\
\hline
\end{tabular}

el teléfono, solicitar la liberación de camas por teléfono, verificar la planilla de vacantes, esperar, reflexionar, anotar, etc. Cabe resaltar también que el $44 \%$ del tiempo total medido se dio en la interacción efectuada entre los enfermeros y médicos -confrontación de saberes de la experiencia, el día a día del paciente en la sala- evolución, interacción medicamentosa y riesgos, con los saberes especializados de los enfermeros y los médicos.

La Figura 2 tiene por objetivo mostrar la actividad continua del técnico de enfermería, en su rutina diaria de anticipaciones, traslados e intercambios de información para gestionar la propia actividad y la de otros, en una búsqueda permanente de eficacia en el trabajo. La recolección de la información mostrada en el gráfico, se hizo por la mañana, en un momento pico de trabajo para gestionar el número de camas, discutir grupalmente las posibles altas, internaciones, nuevas conductas y derivaciones, anticipándose a los ingresos que se producirían en el período de la tarde. La no liberación de camas para la posible entrada de pacientes en el período de la tarde provocaría un obstáculo muy grande al equipo y aumentaría aún más el riesgo en la puerta de entrada por la posibilidad de rechazo del paciente.

Una buena parte del tiempo se utilizó, principalmente, para discutir casos con el equipo de enfermería, anotar datos y compartir información con el equipo médico, además de atender el teléfono. Esa gestión se mostró necesaria para regular el contexto y disminuir la carga de trabajo tanto individual como colectiva por medio de la ampliación de los campos de posibilidades de acción y regulación, lo que puede ser observado en el siguiente fragmento de las notas de campo:
...la técnica Regina revela a Raúl que, luego de haber verificado en el sistema SIGH, no observó en la historia clínica las medidas de glucemia del paciente. En las anotaciones de enfermería ven que la presión arterial (PA) del paciente está en 160/100 mmHg. La médica regresa al puesto de enfermería y los técnicos discuten con ella sobre la pre- sión arterial del paciente. Plantean la necesidad de entrar con medicamentos si la presión sigue alta. El técnico Raúl le dice a la médica: "medique y haga un informe para el servicio sustituto. Ellos deben seguir el proceso. Si es hipertenso y está viniendo de allá, ya debería venir medicado". La médica escucha y decide medicar al paciente.

Se revela aquí la actuación competente como estrategia de gestión en el trabajo. Hubo una apropiación de las normas preexistentes asociadas a la historia y la singularidad de la acción situada basada en valores dimensionables y no dimensionables. Esta gestión no se refleja aquí como individual, sino instaurada colectivamente en las entidades colectivas relativamente pertinentes $(\mathrm{ECRP})^{(33)}$, dimensiona la gestión de camas (dinámica de entrada y salida de pacientes), la calidad de la asistencia y de la clínica a establecer, y de la gestión de la salud y la seguridad. La idea 
de trabajar juntos en la definición de bienes comunes tiene una pertinencia, una significación. Si los valores puestos en discusión tienen adherencia y fuerza para crear entidades pertinentes se puede deducir que la participación de esas personas en los debates, en los campos político y de los valores es coherente. Hay una dialéctica de lo local y de lo global en doble sentido.

Para una actuación competente se deben articular ciertos ingredientes. Estos colectivos de contornos variables, relativamente pertinentes y esenciales, se constituyen sobre el principio de eficacia en el trabajo. Se trata de una creación local de los diferentes protagonistas de la situación, de un cierto modo de "vivir", de construir la vida en el trabajo.

Cuando se observan variaciones y contingencias, aunque algunas soluciones fracasen, se puede comprender que no basta solo con ubicarse en el centro de atención de crisis para gestionar los problemas. Para sacar provecho del medio y actuar de forma competente, es necesario salir del lugar, buscar y confrontar informaciones, hacer Ilamadas telefónicas, dirigirse a la ventanilla de entrada, compartir saberes prácticos y técnicos con otros enfermeros y con el equipo médico, relevar la información del sistema y de las planillas con anotaciones. Anticiparse es necesario para gestionar la actividad, tal como se observa en la siguiente nota de campo:

...el técnico Raúl agarra el teléfono y llama al CMT. Pregunta si la ambulancia ya fue a buscar a los pacientes. Al mismo tiempo, tapa el teléfono y habla con el médico sobre la necesidad de abrir vacantes en el centro de atención de crisis porque, según el técnico, en poco tiempo entrarían más pacientes. Dice: "fui allá afuera y vi muchas personas agitadas, que demandaban entrar". Mira la planilla, mientras les hablaba a los técnicos (dos que estaban en el sector) y al médico. Y continúa: "las camas de las salas ya están todas reservadas por el director" (vacantes reservadas por mandato judicial). Vuelve al teléfono, habla, anota y responde al otro técnico que la ambulancia ya viene y que puede llevar al paciente para el CMT. Corta el teléfono, busca las historias clínicas y las organiza en una secuencia elegida por él y se las entrega al médico que retorna al sector. Luego de la salida del médico, dice: "hay pacientes con indicación de alta, pero como no podemos decidir eso, ya colocamos las historias clínicas en orden según las que consideramos que podrían salir. Así, evalúan a estos pacientes primero y liberan las camas más rápidamente. Es el único modo de dar cuenta de este sector. Si no fuera así, ¿imagina si la historia clínica del paciente de alta fuera el último? Comenzaría a entrar gente sin pasar por el CAC. La experiencia nos hace construir los caminos...

Tanto la Figura 2 como los fragmentos presentados muestran el trabajo específico, imprevisible e imprescriptible, de una entidad colectiva relativamente pertinente (ECRP). Si el colectivo no funcionara de forma armónica, sea por las faltas y/o la rotación de los trabajadores, y hubiera "desincronización" y fallas, aparecerían riesgos en el trabajo, y el hospital, los trabajadores y los pacientes pagarian el costo de eso. La construcción de competencias y su atualización es una necesidad fundamental de la gestión de riesgos en este contexto.

El origen de las complicaciones (atraso de la ambulancia para transferir pacientes, llegada de diversos casos nuevos, etc.) y de todos otros tipos de dificultades puede ser la consecuencia de algo muy distante en el tiempo y en el espacio. Según Schwartz y Durrive ${ }^{(33)}$, "una entidad colectiva es también así: para conducir una acción a su ritmo en condiciones casi correctas, no se puede circunscribir jamás, con anticipación, en el espacio y en el tiempo, aquello que es pertinente como zona de intercambio, de comunicación".

Estas acciones realizadas colectivamente en el contexto del centro de atención de crisis son una reinvención permanente. Es una verdadera sinfonía sin maestro, o sea, cada uno toca su partitura (sabe lo que tiene que hacer) pero, al mismo tiempo, es necesario que 
la partitura sea sincrónica, por lo tanto, que tenga marcaciones para que se sepa cuándo entra cada uno, ya que no hay un director.

\section{DISCUSIÓN}

El hospital también ha pasado por transformaciones: se ha tornado cada vez menos un lugar de permanencia $y$, cada vez más, un lugar de paso, principalmente, en las urgencias y emergencias ${ }^{(8)}$. El paciente que llega en crisis es asistido, controlado y derivado para su seguimiento en los servicios sustitutos de la red de salud mental ${ }^{(21,39)}$. Por lo tanto, este hospital integra y refuerza la red.

Según el gestor de la institución, "el CAC es fundamental para la atención de urgencia y para evaluar la posibilidad de internación y/o vinculación de pacientes a los servicios sustitutos luego del período de crisis". Según el mismo gestor, para trabajar en el centro de atención de crisis se necesita una experticia o un cuerpo de conocimientos históricos para lidiar, en ese contexto, con el riesgo inherente a la actividad. Y afirmó: "sin el CAC y el relevamiento del riesgo en la puerta de entrada, el número y la gravedad de las agresiones aumentaría absurdamente en las salas". Esta afirmación va al encuentro de la propia noción de riesgo ${ }^{(34)}$, la cual se encuentra retenida entre dos tipos de constataciones: "por un lado, una hipertrofia de normas de seguridad definidas antes de la actividad; por otro, el saber-hacer prudente que se constituye casi en la clandestinidad del transcurso de las actividades".

Un técnico de enfermería de la instituición relata la dramática situación de lidiar con un paciente:

El problema principal es el siguiente: llega el paciente drogado, el muchacho fumó tal y tal. El paciente no acepta quedarse de ninguna manera. Entonces nos vemos obligados a usar la fuerza ¿entendés? Nos vemos obligados a usar la fuerza física, no hay otra forma. Ahora, uno de los mayores problemas que se generan principalmente entre nosotros y el paciente es el siguiente: el médico va y evalúa al paciente, allí e indica internación. Algunos tienen una postura correcta, se acercan al paciente y le dicen: "vas a quedar internado", porque aceptándolo o no si tenemos que hacerlo, lo vamos a agarrar. Muchos médicos no comunican eso al paciente, lo dejan para que lo comunique la gente de enfermería, lo que considero totalmente equivocado ¿entendés? (Técnico de enfermería 1, Centro de atención de crisis).

En el trabajo, se da continuamente un debate entre las normas preexistentes -saberes relativamente estabilizados y protocolares y saberes de la experiencia de los trabajadores-y las normas internas. A partir de ese debate de normas, se hacen elecciones, no siempre conscientes ni sustentadas por los valores. La renormalización se da cuando se produce una ruptura entre los valores que permean la organización y, consecuentemente, la norma antecedente y los valores de los trabajadores.

En la institución analizada, en la última década, se produjo un cambio en el perfil de los pacientes que provocó un aumento en el riesgo potencial de agresión en la puerta de entrada, tal como lo relata un técnico de enfermería:

...esto se transformó en un penal, jesa es la verdad! La mayoría de nuestros pacientes son "drogadictos", alcohólicos y hay muchos pacientes judicializados; pacientes con muertes en sus espaldas, pacientes que roban, que hacen atrocidades. Entonces, aquí en la urgencia la amenaza de muerte es constante, ¿entendés? ¡Constante en serio! Pacientes que amenazan con que nos van a matar... O cada uno se genera estrategias para lidiar con eso o muere. Otra alternativa es irse antes, lo que es común aquí para los novatos. (Técnico de enfermería 3, Centro de atención de crisis).

En el contexto en cuestión, los cambios en el perfil de los pacientes exigen una reconfiguración de las prácticas y de las competencias ${ }^{(40)}$ 
frente a la especificidad de la asistencia psiquiátrica a los usuarios de drogas, donde se registran comúnmente reacciones de violencia.

Estos debates y negociaciones, incluso cuando la decisión es individual, son siempre colectivos. Si la gestión del trabajo implica la gestión de múltiples gestiones, vale aquí resaltar que no hay igualdad de condiciones en ese debate y la negociación de eficacias. Las relaciones de poder-saber establecidas influyen directamente en las decisiones en un campo de disputa complejo. El técnico de enfermería comparte su saber con el colectivo, anticipa, gestiona los riesgos y la asistencia, discute, opina, confronta; sin embargo, la decisión final y las conductas asumidas le caben al médico.

Estos modos de organización de los servicios y de los equipos de salud mental, aún fuertemente centrados en la figura del médico, fueron también mencionados en el estudio de Ramminger y Brito ${ }^{(41)}$ en un centro de atención psicosocial (CAPS) del interior de Río de Janeiro. Según las autoras, en el servicio citado, el médico ocupa un papel central, cuya función es la medicalización. Ante la imposibilidad de atención a las crisis (brotes) realiza muchas veces "altas administrativas", sin carácter terapéutico, sino esencialmente punitivas, con juicios morales disfrazados de argumentación clínica, derivaciones sumarias para internación y la "docilización" a través del encarcelamiento químico. Otros estudios ${ }^{(42,43,44)}$ también revelan un cuadro parecido.

Según Foucault ${ }^{(38)}$, ante la hipótesis de anexar la locura a la enfermedad mental, la acción de la psiquiatría se revela como una policía de los locos revestida de filantropía. Este mismo autor ${ }^{(45)}$, abocado a las relaciones entre el sujeto y las prácticas de poder, refuerza que "las grandes reformas, no solo de la práctica psiquiátrica, sino del pensamiento psiquiátrico, giran en torno de esta relación de poder, constituyen tentativas para desplazarlo, enmascararlo, eliminarlo y anularlo"(46).

Para Arendt ${ }^{(47)}$, los fenómenos de la violencia y del poder se ubican en el ámbito político de la actuación humana. En cierto sentido, se puede decir que cualquier acción organizada obliga a los operadores a desarroIlar el potencial de gestión de las competencias, o sea, percibir y traducir los elementos de la situación -competencias de traducción de la acción en curso- y hacer emerger los saberes para la acción/reacción.

En la dinámica colectiva del trabajo, a partir de la entrada del paciente en el flujo hospitalario, las competencias de explicitación, de intervención y de evaluación ${ }^{(25)}$ de los técnicos asociadas a las competencias del equipo técnico especializado son recursos fundamentales para la evaluación del riesgo de violencia y la definición de estrategias de abordaje ante la inminencia del riesgo.

En este contexto, el riesgo sería la réplica lógica de una doble imposibilidad vinculada a la naturaleza de la actividad humana, más allá de la forma y la organización histórica que asuma: la imposibilidad de neutralización de las singularidades en las actividades humanas y de anticipación total de los elementos constitutivos del proceso de la actividad ${ }^{(34)}$. Es en este sentido que las actividades humanas son el lugar de compromiso de sus autores.

Como el siguiente relato de un técnico de enfermería del centro de atención de crisis, respecto de la importancia del colectivo y la experiencia necesaria para llevar a cabo las tareas en aquel sector:

Ellos suman, suman mucho. Y cuando es necesario que venga alguien de otra unidad para actuar en el CAC por falta de profesionales, porque sucedió algo, alguien tuvo que irse, tuvo que ir al médico, sentís drásticamente cómo el servicio se complica. Porque la persona no tiene experiencia, ¡no tiene! No es que no sean dinámicos, sino que el dinamismo del CAC es diferente. Ya sabés, el paciente llega y uno ya comienza a controlar signos vitales, otro ya lo manda para el baño. El que lo deriva al baño ya va agarrando sus pertenencias y separándolas; otro ya va haciendo la admisión en la computadora. Tiene que ser una cosa así, jágil! Porque si un enfermero lo quiere hacer todo solo y llega 
otro paciente, ¿y ahí? Ahí se va a generar revuelo, no podés dejar que el servicio se altere. (Técnico de enfermería 4, Centro de atención de crisis).

$\mathrm{Y}$, respecto al riesgo de violencia, mencionó:

Creo que cuando llegamos a la mañana tenemos que pegar una frenada. Ya llegás esperando que todo sea imprevisible, violento, que te agredan. Pasa el tiempo, y no está sucediendo eso, estas demandas, y comenzás a frenar un poco más, Ilegás con una cierta agitación al entrar en el clima del CAC. La violencia es intensa. El colectivo tiene que aparecer. Estás siempre en demanda, en alerta. Cuando está todo tranquilo, tenés que ir frenando, pero eso no quiere decir que esa imprevisibilidad no suceda, eso ya lo tenés presente. En cualquier momento, el interruptor se va a encender y la demanda de todos los profesionales va a ocurrir. Ese engranaje, ¿no? Va comenzar a funcionar y es como si fuese un engranaje, todo va a tener que encajar ahí, y todos ya conocen su lugar en ese momento. (Técnico de enfermería 7, Centro de atención de crisis).

Al preguntarle qué significaba "ir frenando", reveló: "ir frenando no es quedarse parado. Es estar alerta, vigilante, anticiparse para soportar los problemas. Es tener una mirada sobre la totalidad. Es entender lo que dice el compañero sin que hable. Es saber qué equipo va a actuar. $\mathrm{Si}$ el equipo médico es compañero o impositivo. Es hacer".

Para el diagnóstico de este contexto fue necesaria una postura de rigor y humildad para valorar el saber de los trabajadores sobre su propia actividad y, al mismo tiempo, plantear un diálogo con los saberes instituidos, lo que de cierta forma, hizo emerger "lo que se hace y no puede ser dicho. Lo que se dice que se debe hacer y no puede hacerse"(48).

La construcción de competencias y su actualización es una necesidad fundamental de gestión de riesgos de violencia en este contexto. Las soluciones posibles de las disfunciones locales y el riesgo de violencia no están en el aumento de la reglamentación y de medios de protección local (necesarios muchas veces para ciertos tipos de riesgo), sino en la definición de formas organizacionales que hagan emerger la capacidad de gestión de los protagonistas del trabajo en el curso de la actividad.

\section{CONSIDERACIONES FINALES}

El abordaje analizado del riesgo se restringía a los riesgos profesionales. Sin embargo, la relación entre el riesgo y la actividad humana no permite restringir el abordaje solo al riesgo. En el trabajo hay una dimensión enigmática, por lo que los riesgos locales se relacionan con las negociaciones entre las personas, los colectivos y las "condiciones de trabajo", siempre más o menos retrabajadas.

En el centro de atención de crisis y, por consiguiente, en el hospital se observó, por un lado, una hipertrofia de normas, incluso de seguridad, definidas antes de la actividad $y$, por otro, un saber-hacer construido e instituido casi en la clandestinidad del transcurso de las actividades: la infracción se colocaba allí como condición necesaria para la producción de un saber-hacer prudente, útil para la eficacia y la salud en el trabajo.

La instrumentalidad de las dimensiones del cuidado establecidas en el centro de atención de crisis refuerza el abordaje utilizado ante el riesgo potencial. La ampliación de los campos de posibilidad de gestión del riesgo depende de las condiciones organizacionales para actualizar las competencias del colectivo, en el que los técnicos de enfermería ocupan un lugar fundamental. Por lo tanto, la valoración del trabajo de esos técnicos, que implica, la estabilidad de los vínculos, la equidad de la carga horaria y de la remuneración del conjunto de los técnicos son procedimientos esenciales. 


\section{REFERENCIAS BIBLIOGRÁFICAS}

1. Mendes DP. O agir competente como estratégia de gestão do risco de violencia no trabalho: o ponto de vista da atividade humana de trabalho dos técnicos de enfermagem de uma instituição pública psiquiátrica. [Tesis de doutorado]. Belo Horizonte: Universidade Federal de Minas Gerais; 2014.

2. Maciel EMGS, Telles FSP. Ensaio sobre a relação epistemológica entre probabilidade e método científico. Cadernos de Saúde Pública. 2000;16(2):487497.

3. Areosa J. O risco no âmbito da teoria social. En: VI Congresso Português de Sociologia; Lisboa; 2008.

4. Gondim GMM. Do conceito de risco ao da precaução: entre determinismos e incertezas. En: Fonseca AF, Corbo AD. O território e o processo saúde-doença. Rio de Janeiro: EPSJV/Fiocruz; 2007. p. 87-119.

5. Mazet C, Guillermain H. Concepts de base. En: Amalberti R, Mosneron-Dupin F, (eds.). Facteurs humains et fiabilité. Toulose: Octares éditions; 1997. p. 16-19.

6. Goetsch DL. Occupational safety and health for technologists, engineers, and managers. Toronto: Pearson Prentice Hall; 2006.

7. Vieira MA. Autoconfrontação e análise da atividade. En: Figueiredo $M$, Athayde $M$, Brito J, Alvarez D, (orgs.). Labirintos do trabalho: Interrogações e olhares sobre o trabalho vivo. Rio de Janeiro: DP\&A; 2004. p. 161-187.

8. Mendes D, Silva A, Barbosa C, Lima J, Oliveira $\mathrm{M}$, Matos V. Um olhar sobre a atividade de trabalho de auxiliares e técnicos de enfermagem de uma instituição psiquiátrica: em busca de transformações. En: XV Congresso Brasileiro de Ergonomia; 2008; Bahia.

9. Oliveira A, Alessi N. Trabalho de enfermagem em saúde mental: contradições e potencialidades atuais. Revista Latino-Americana de Enfermagem. 2003;11(3):333-340.

10. Almeida MCP, Mello DF, Neves LAS. O trabalho de enfermagem e sua articulação com o processo de trabalho em saúde coletiva: rede básica de saúde em Ribeirão Preto. Revista Brasileira de Enfermagem. 1991;44(2-3):64-75.

11. Oliveira BRG, Murofuse NT. Acidentes de trabalho e doença ocupacional: estudo sobre o conhecimento do trabalhador hospitalar dos riscos à saúde de seu trabalho. Revista Latino-Americana de Enfermagem. 2001;9(1):109-115.
12. Manetti ML, Marziale MHP, Robazzi ML. Revisando os fatores psicossociais do trabalho de enfermagem. Revista da Rede de Enfermagem do Nordeste. 2008;9(1):111-119.

13. Sulzbacher E, Fontana RT. Concepções da equipe de enfermagem sobre a exposição a riscos físicos e químicos no ambiente hospitalar. Revista Brasileira de Enfermagem. 2013;66(1):25-30.

14. Fernandes MA, Marziale MHP. Occupational risks and illness among mental health workers. Acta Paulista de Enfermagem. 2014;27(6):539-547.

15. Guerra PC, Oliveira NF, Terreri MTRA, Len CA. Sleep, quality of life and mood of nursing professionals of pediatric intensive care units. Revista da Escola de Enfermagem da USP. 2016;50(2):277283.

16. Haenscke Senna M, Lima Pestana A, Marcellino de Melo Lanzoni G, Lorenzini Erdmann A, Schlindwein Meirelles BH. Seguridad del trabajador en la manipulación de antineoplásicos. Avances en Enfermería. 2013;XXXI(1):141-158.

17. Vasconcelos SC, Souza SL, Sougey EB, Ribeiro ECO, Nascimento JJC, Formiga MB, Ventura LBS, Lima MDC, Silva AO. Nursing staff members mental's health and factors associated with the work process: an integrative review. Clinical Practice and Epidemiology in Mental Health. 2016;12:167-176.

18. Pelissier C, Fontana L, Fort E, Agard JP, Couprie F, Delaygue B, Glerant V, Perrier C, Sellier B, Vohito M, Charbotel B. Occupational risk factors for upper-limb and neck musculoskeletal disorder among health-care staff in nursing homes for the elderly in France. Industrial Health. 2014;52(4):334-346.

19. Marziale MHP, Rocha FLR, Robazzi MLCC, Cenzi CM, Santos HEC, Trovó MEM. Organizational influence on the occurrence of work accidents involving exposure to biological material. Revista Latino-Americana de Enfermagem. 2013;21:199-206.

20. Manetti ML, Marziale MHP, Robazzi MLCC. Revisando os fatores psicossociais do trabalho de enfermagem. Revista da Rede de Enfermagem do Nordeste. 2008;9(1):111-119.

21. Mendes DP, Moraes GFS, Mendes JCL. Análise da gestão de risco no trabalho de enfermagem em uma instituição psiquiátrica. Trabalho \& Educação. 2011;20(1):73-84.

22. Bourrier $\mathrm{M}$, Bieder $\mathrm{C}$. Trapping safety into rules: how desirable or avoidable is proceduralization? Farnhan: Ashgate Publishing Limited; 2013. 
23. Boyé M, Robert G. Gérer les compétences dans les services publics. Paris: Les Éditions d'Organisation; 1994.

24. Leplat J, Montmollin M, (orgs.). Les competences en ergonomie. Toulose: Octarès Éditions; 2001.

25. Terssac G. Compétences et travail: compétences d'explicitation, d'intervention et d'évaliation. En: Leplat J, Montmollin M, (orgs.). Les compétences en ergonomie. Toulouse: Octarès Éditions. 2001. p. $95-100$.

26. Cunha DM. Notas conceituais sobre atividade e corpo-si na abordagem ergológica do trabalho. En: 30a Reunião anual da ANPED; Caxambu; 2007.

27. Schwartz Y. Ergonomie, philosophie et exterritorialité. En: Daniellou F, (org.). L'ergonomie en quête de ses principes: débats épistémologiques. Toulouse: Octarès Éditions; 1996. p. 141-182.

28. Schwartz Y, Durrive L, (orgs.) Trabalho e ergologia: conversas sobre atividade humana. Niterói: EdUFF; 2010

29. Athayde M, Brito J. Ergologia e clínica do trabalho. En: Bendassolli PF, Soboll LA, organizadores. Clínicas do Trabalho: novas perspectivas para compreensão do trabalho na atualidade. São Paulo: Editora Atlas; 2011. p. 258-281.

30. Schwartz Y. As linhas de desenvolvimento específicas da abordagem ergológica. Belo Horizonte: UFMG; 2013.

31. Schwartz Y. O enigma do trabalho: riscos profissionais e riscos do trabalho. En: Bianco MF, (org.). Competências e gestão: dialogando com o trabalho e decifrando suas conexões. Vitória: Proex, UFES; 2014. p. 59-70.

32. Echternacht E. Atividade humana e gestão da saúde no trabalho: elementos para reflexão a partir da abordagem ergológica. Laboreal. 2008;4(1):46-55

33. Schwartz Y, Durrive L. Trabalho e ergologia: conversas sobre atividade humana. Niterói: EdUFF; 2010 .

34. Nouroudine A. Risco e atividades humanas: acerca da possível positividade aí presente. En: Figueiredo M, Athayde M, Brito J, Alvarez D, organizadores. Labirintos do trabalho: interrogações e olhares sobre o trabalho vivo. Rio de Janeiro: DP\&A; 2004. p. 37-62.

35. Schwartz Y. De I'inconfort intellectuel ou comment penser lês activités humaines. En: Le pa- radigme ergologique ou un métier de philosophe. Toulose: Octarès Editions; 2001.

36. Guérin F, Kerguelen A, Laville A, Daniellou F, Duraffourg J. Compreender o trabalho para transformá-lo: a prática da ergonomia. São Paulo: Edgard Blücher; 2001.

37. Schwartz Y. A comunidade científica ampliada e o regime de produção de saberes. Revista Trabalho \& Educação. 2000;7(1):38-46.

38. Foucault M. História da loucura na idade clássica. São Paulo: Perspectiva; 1978.

39. Mendes DP, Echternacht EH. Gestão do risco de violência em hospitais públicos psiquiátricos e as competências dos técnicos de enfermagem. En: III Congresso Latino-Americano de Ergonomia; XVI Congresso Brasileiro de Ergonomia; 2010; Rio de Janeiro.

40. Rogalski J, Rabardel P, Janin R. L'identification des dimensions des changements de technologie, d'organisation du travail et d'évolution des compétences. En: Leplat J, Montmollin M, (dirs.). Les compétences en ergonomie. Toulouse: Octarès Éditions; 2001. p. 95-100.

41. Ramminger T, Brito JC. "Cada CAPS é um CAPS": uma coanálise dos recursos, meios e normas presentes nas atividades dos trabalhadores de Saúde Mental. Psicologia \& Sociedade. 2011;23:150-160.

42. Sznelwar LI, Mascia F, Montedo U, Brunoro C, Abrahao J. Análise ergonômica do trabalho: ação ergonômica. En: Lancman S, Costa ACF, (orgs.). Políticas públicas e processos de trabalho em saúde mental. Brasília: Paralelo 15; 2008. p. 129-174.

43. Silva ER. A atividade de trabalho do psiquiatra no CAPS-centro de atenção psicossocial: pois é José. [Dissertação de Mestrado]. Belo Horizonte: Universidade Federal de Minas Gerais; 2010.

44. Almeida DT. Análise do trabalho de uma equipe multiprofissional em um centro psicossocial álcool e drogas. [Dissertação de Mestrado]. Belo Horizonte: Universidade Federal de Minas Gerais; 2012.

45. Foucault M. Estratégia, poder-saber. Rio de Janeiro: Forense Universitária; 2006

46. Foucault M. Microfísica do poder. Rio de Janeiro: Graal; 2009.

47. Arendt H. Sobre a violência. Rio de Janeiro: Civilização Brasileira; 2010. 
48. Lhuilier D. A invisibilidade do trabalho real e a opacidade das relações saúde-trabalho. Trabalho \& Educação. 2012;21(1):13-38.

\section{FORMA DE CITAR}

Mendes DP, Cunha DM. La opacidad del trabajo de enfermería y las configuraciones del riesgo. Salud Colectiva. 2018;14(4):725-742. doi: 10.18294/sc.2018.1349.

Recibido: 14 de marzo de 2017 | Versión final: 14 de enero de 2018 | Aprobado: 3 de mayo de 2018

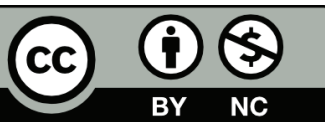

Este obra está bajo una licencia de Creative Commons Reconocimiento-NoComercial 4.0

Internacional. Reconocimiento - Permite copiar, distribuir y comunicar públicamente la obra.

A cambio, se debe reconocer y citar al autor original. No Comercial - Esta obra no puede ser

utilizada con finalidades comerciales, a menos que se obtenga el permiso.

http://dx.doi.org/10.18294/sc.2018.1349

Este artículo fue traducido del portugués por Viviana Martinovich. 